\title{
Simulation Based Analysis of Bee Swarm Inspired Hybrid Routing Protocol Parameters Using Realistic Mobility Model In Vehicular Ad-Hoc Networks
}

\author{
Kamaljeet Kaur \\ Department of Computer Science and Engineering \\ G.G.S.C.E.T, Guru Kashi University \\ Talwandi Sabo, India
}

\begin{abstract}
Vehicular Ad-hoc Networks (VANET's) are basically emanated from Mobile Ad hoc networks (MANET's) in which vehicles act as the mobile nodes, the nodes are vehicles on the road and mobility of these vehicles are very high. The main objective of VANET is to enhance the safety and amenity of road users. It provides intelligent transportation services in vehicles with the automobile equipment to communicate and co-ordinates with other vehicles in the same network that informs the driver's about the road status, unseen obstacles, internet access and other necessary travel service information's. The evaluation of vehicular ad hoc networks applications in based on the simulations. A Realistic Mobility model is a basic component for VANET simulation that ensures that conclusion drawn from simulation experiments will carry through to real deployments. This paper attempts to evaluate the performance of a Bee swarm inspired Hybrid routing protocol for vehicular ad hoc network, that protocol should be tested under a realistic condition including, representative data traffic models, and the realistic movement of the mobile nodes which are the vehicles. In VANET the simulation of Realistic mobility model has been generated using SUMO and MOVE software and network simulation has been performed using NS2 simulator, we conducted performance evaluation based on certain metric parameters such as packet delivery ratio, end-to-end delay and normalized overhead ratio.
\end{abstract}

Keywords: Vehicular Ad hoc Network (VANET), Bee swarm routing protocol, Genetic Algorithm, Realistic mobility model, SUMO, MOVE, NS-2, Simulations.

\section{INTRODUCTION}

Vehicular Ad hoc Network (VANET) is a new revolutionary phase of automotive industry. Today, a vehicle is not just a thermo mechanical machine with few electronic devices; rather, recent advancement in wireless communication technologies has brought a major transition of vehicles from a simple moving engine to an intelligent system carrier.[1] The components of VANET mostly resembles the MANET features like as the techniques of self-configuring, selfmanagement and autonomous wireless network. A vehicular ad-hoc network is a technology that uses moving vehicles as nodes in a network to establish a arbitrary mobile network. VANET provides communication between adjacent vehicles and between vehicles and nearby fixed point and offer some intelligent activities. It is an intelligent network of vehicles, called Intelligent Transportation System (ITS) which is used to ensure the security services of driver assistance and comfort of road users. Intelligent Transportation System include all types of communication in vehicles, between vehicles-to-vehicles (V2V) and Vehicle-to-Infrastructure (V2I).[2] The basic target of these communications is to increase the road safety, provide the better driving surroundings and cautioning messages need to be sent to the vehicles for different functions through the inter-vehicle communications.

VANET is a subset of Mobile Ad hoc Network (MANET) which consists of number of vehicles with the ability to communicate with each other, they transmit information via radio in order to enhance the security and efficiency of transportation system, and allow internet access to the users. It is supposed that each vehicle has a wireless communication equipment to provide ad hoc connectivity. Such networks comprise of sensors and On Board Units (OBU) installed in the car as well as Road Side Unit (RSU). The data collected from sensors on the vehicles can be displayed to the drivers and sent to the RSU or even broadcasted to other vehicles depending on its nature and importance.[3]

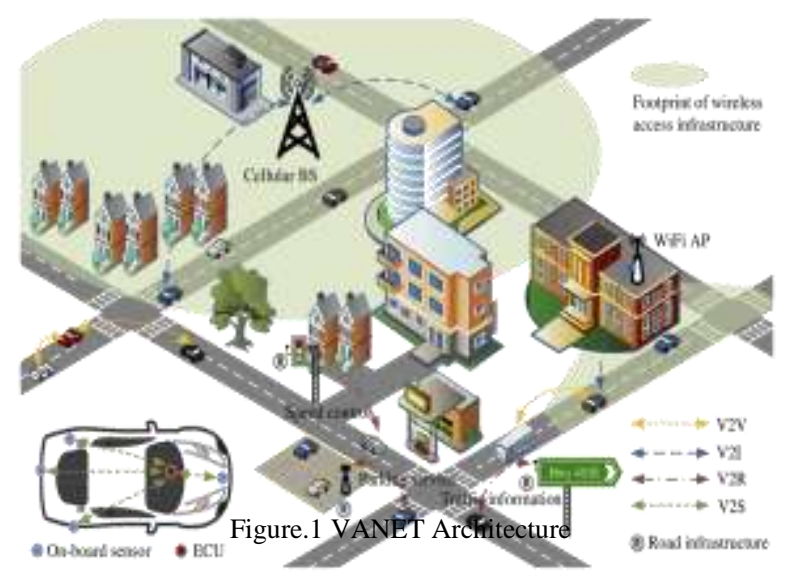

One of the main challenges in the full deployment of VANET is the testing of routing protocols and algorithms because vehicular ad hoc networks topologies are very different by 
nature as it changes very frequently and rapidly, basically it depends on the nature of movement of vehicles, scopes and scale of VANET networks. So, effectively design routing protocol with a more intelligent use of resources is a good way to cover these problems and able to improve the performance of the transmission and dissemination of information which are made according to the routing protocols.

In this paper, we proposed a Hybrid Bee Swarm Routing Protocol which is developed by using Bee swarm routing protocol as well as Genetic Algorithm simultaneously that can efficiently handle the variations of network parameters to keep its performance constant over network changes. For testing and evaluating this proposed protocol we used Realistic Mobility Model. The high mobility of nodes in VANET makes mobility model selection one of the most important parameters when evaluating any protocol. Mobility models helps to determine the location of nodes in the topology at any given instant, which strongly affects network connectivity and throughput.[4] The performance of the proposed protocol has been studied using simulation tools SUMO and MOVE and also Network Simulator(NS2).

\section{RELATED WORK}

Vehicular Ad hoc Network (VANET) present various services to the road users to communicate and transmit information with each other to help out the vehicle drivers to avert any kind of critical situations with providing secure and safely environment. For the transaction of data information between two entities in a network we need to use an efficient routing protocol to manage the performances of vehicles at different network parameters. In this paper, we introduced a Bee Swarm Hybrid Routing Protocol for the improvement of the routing efficiency, to keep its performance constant whenever the network parameters changes. The proposed protocol is basically a combination of two techniques that is Bee swarm routing protocol and Genetic Algorithm. Bee Swarm routing protocol is describe the intelligent foraging nature of honey bee swarm which is consists of three groups: packers, scouts and foragers. Honey bees use distinct dancing techniques for communication which is performed by the scout bees like as waggle dancing and round dancing. If the food is closure to the hive, bees dance in circular motion that is known as round dancing and the waggle dance is the dance when bee repeated a same move in a form of number eight which implies the distance and the direction of food. This protocol is able to vigorously find out multiple paths between source and destination, and also helps to distribute traffic across them.

Other technique used in proposed system is the Genetic algorithm. Genetic Algorithm is heuristic technique that implies the methods to detect the ideal solutions which is based on the assumptions of natural evolution and genetics. In this proposed system we use these two techniques simultaneously in order to establish the hybrid algorithm. In this algorithm the individual (is set of solutions available for particular problem) refers to nodes that are participating in the route from source to destination, Population indicate the group of individuals that are selected as participant for the routing process from source to destination. Fitness function is used to identify the compatible solution for the problem; if the evaluated individuals execute their functions in appropriate way then they got chances to be selected as a fittest candidate node for routing from source to destination.

This proposed algorithm will provide the reliable and efficient routes from the source node to the destination node with better performance results. It is not possible for directly implementation of this protocol due to the cost and complexity. So, the simulation becomes the tool of choice to evaluate these solutions. In this paper, we used a Realistic Mobility Model for the simulation of the evaluated routing protocol which characterized the real world region. These Mobility model represents the flow diagram of mobile users, including its location, speed and acceleration over time.[2] In Realistic Mobility Model the proposed protocol should be tested and evaluated in realistic conditions like as the movement of the nodes which are vehicles and assure that the achieved results will carry through the real world zone.

\section{SIMULATION METHODOLOGY}

The Simulation Methodology is a very crucial way to formulating the realistic evaluation of the network; it provides the manifest idea of the involved research. So, choosing the appropriate methodology is very necessary as it will enhance the performance of this system. In this paper, we are going to use Simulator for Urban mobility software (SUMO) and MOVE (Mobility model generator for Vehicular networks) and also Network Simulator (NS2.35) software to run simulations. By using SUMO software, we created a Realistic Mobility Model for Vehicular Ad hoc Networks (VANET'S).

\subsection{Mobility Model Generator for Vehicular Networks (MOVE)}

Mobility model generator for vehicular networks (MOVE) is software to facilitate users to rapidly generate realistic mobility model for VANET simulations. MOVE is a javabased application built on SUMO with a facility of GUI. It provides an environment that allows users to quickly pinpoint incorrect details and manage details overhead. MOVE is built on the top of an open source micro-traffic simulator SUMO.[6] The output of MOVE is a mobility trace file that consists the information of realistic vehicle movements which can be instantly used by the network simulators such as NS2 and qualnet. It allows users to generate realistic mobility models by two approaches: interfacing with real world data map and other one is with Google earth.

\subsection{Simulation of Urban Mobility (SUMO)}

Simulation of Urban Mobility (SUMO) is an open source traffic simulation package including net import and demand modeling components. SUMO helps to investigate several topics e.g. route choice and traffic light algorithm or simulating vehicular communication. Therefore the framework is used in different projects to simulate automatic driving or traffic management strategies. [7] It allows the user to build a customized road topology, in addition to import the different readymade map formats of many cities and towns of the world. The map area which is to be simulated is exported from OpenStreetMap(OSM) which is saved as .osm file. This .osm file converted into .net.xml file. The route file and 
.net.xml file then transformed into sumo trace.xml file using the configuration file of sumo, this file is changed into NS2 file using trace Exporter.py of SUMO which is used for the simulation of mobility model nodes of VANET.

\subsection{Network Simulator (NS 2.35)}

Network simulation is a widely used tool to simulate the behavior of wired and wireless networks. It is a technique where a program models the behavior of a network either by calculating the interaction between the different network entities. NS is a discrete event simulator targeted at networking research. Ns provides substantial support for simulation of TCP, routing and multicast protocols over wired and wireless (local and satellite) networks.[8] The scripting of NS is written in $\mathrm{C}++$, with an Otcl interpreter. In our case 2.35 version of network simulator is used which is commonly called as NS2.

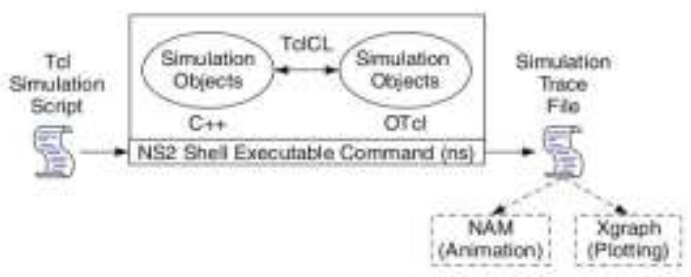

Figure.2 Basic Architecture of NS

\section{SIMULATION SETUP}

The VANET network is simulated using SUMO and MOVE and also NS -2.35 to analyze the performance of the proposed protocol parameters. The Simulation parameters that are used in this simulation given below in the following table:

Table I : Simulation Setup

\begin{tabular}{|c|c|c|}
\hline S.No. & Parameters & Values \\
\hline 1 & NS version & ns-allinone-2.35 \\
\hline 2 & MOVE version & 0.12 .3 \\
\hline 3 & SUMO version & Wireless \\
\hline 4 & Channel & Wireless physical \\
\hline 5 & Network Interface & Drop tail/Priority queue \\
\hline 6 & Queue & Omni-Antenna \\
\hline 7 & Antenna Type & 300 seconds \\
\hline 8 & Simulation Time & 40 (km/hr) \\
\hline 9 & Speed & Mac 802_11 \\
\hline 10 & Mac Type & Two ray Propagation \\
\hline 11 & Interface Queue Length & \\
\hline 12 & Propagation Model & \\
\hline
\end{tabular}

\begin{tabular}{|c|c|c|}
\hline 13 & Routing Protocols & $\begin{array}{c}\text { AODV, Bee Swarm } \\
\text { routing protocol, } \\
\text { Modified hybrid routing } \\
\text { protocol }\end{array}$ \\
\hline 14 & Number of Nodes & 40,100 \\
\hline 15 & Mobility Model & Realistic Mobility Model \\
\hline
\end{tabular}

\section{SIMULATION RESULTS AND ANALYSIS}

In this paper, we basically generated Realistic Mobility Model and presented the analysis of the proposed hybrid routing protocol using the performance metrics such as Packet delivery ratio, end-to-end delay and normalized overhead ratio.

For the research of the proposed system we generated realistic mobility model by using SUMO and MOVE. To generate Realistic model we have to create a road map of the research area and its vehicle movement. These model screenshots are shows up in the given below figures:

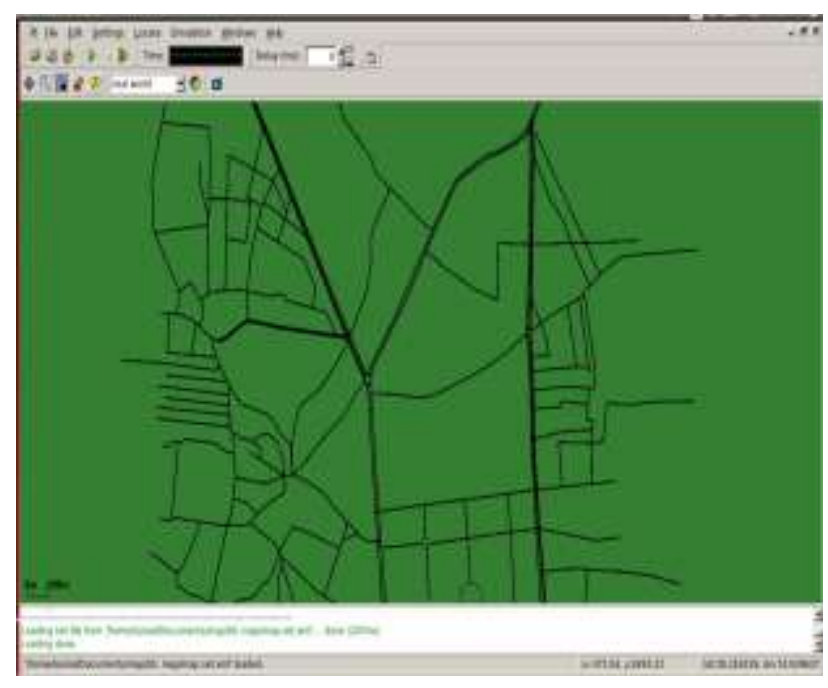

Figure.3 Road Map visualization

The movement of vehicles can be generated automatically or manually by using the vehicle movement editor and we can also specified distinct vehicle route properties by movement editor. 


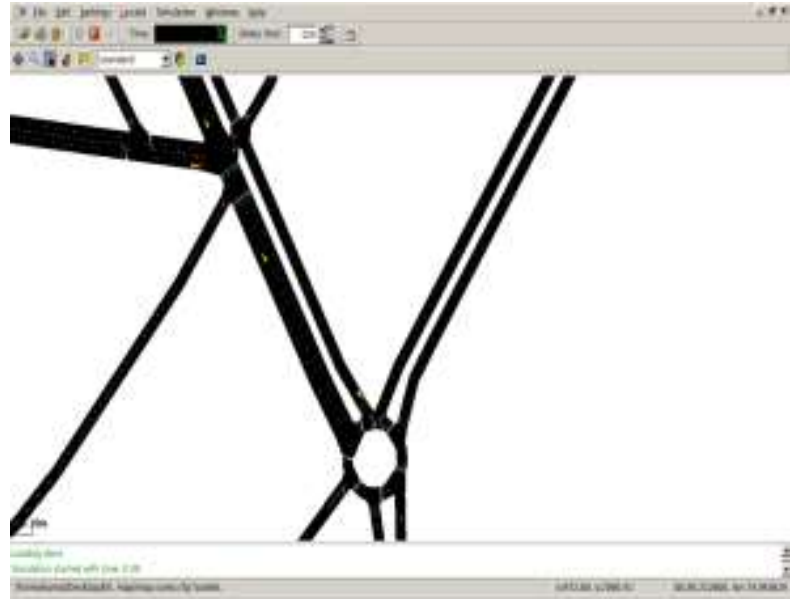

Figure.4 Vehicle Movement on road

\subsection{NETWORK SIMULATIONS}

The screenshots of simulation in Ns-2 nam trace file shown below in the figure: 5-6 which consists 40 and 100 nodes in the network. In this it captured the node movement and communication between other active vehicles.

\section{For 40 Nodes}

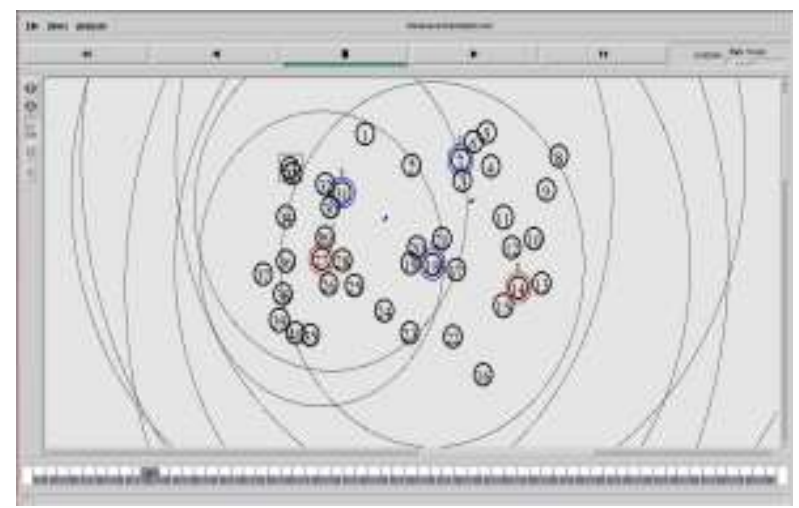

Figure.5 Network scenario for 40 nodes of the vehicular network with random distribution

\section{For 100 Nodes}

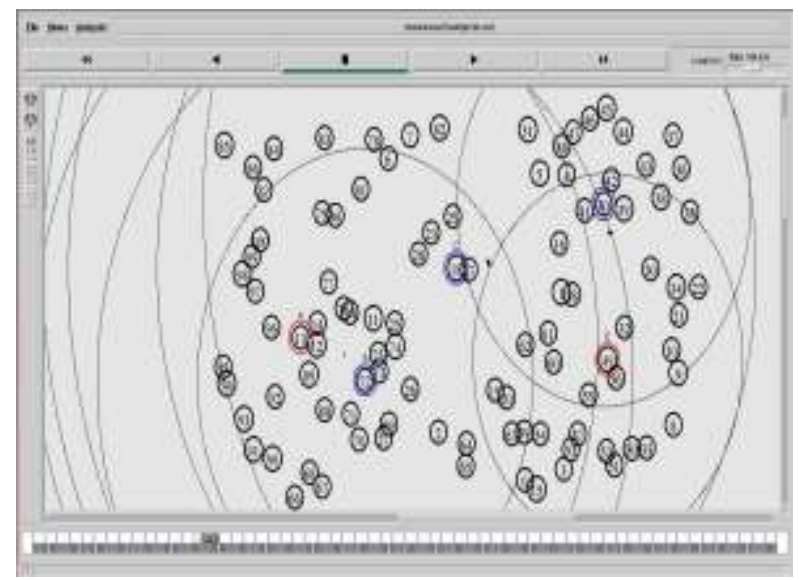

Figure.6 Network scenario for 100 nodes corresponding to the high traffic density

\subsection{PERFORMANCE ANALYSIS}

The proposed system is simulated and tested for 40,100 nodes to evaluate the performance parameters. Here we analyzed some of the performance metrics such as Packet Delivery Ratio, End-to-End delay, Normalized Overhead Ratio which are shown in the following line graphs.

\subsubsection{PACKET DELIVERY RATIO}

The Packet delivery ratio in this simulation process is defined as the ratio of the number of data packet $s$ sent to the destination by constant bit rate sources. This illustrates the level of delivered data to the destination.

$\sum$ Number of packet receive / $\sum$ Number of packet send

For 40 Nodes

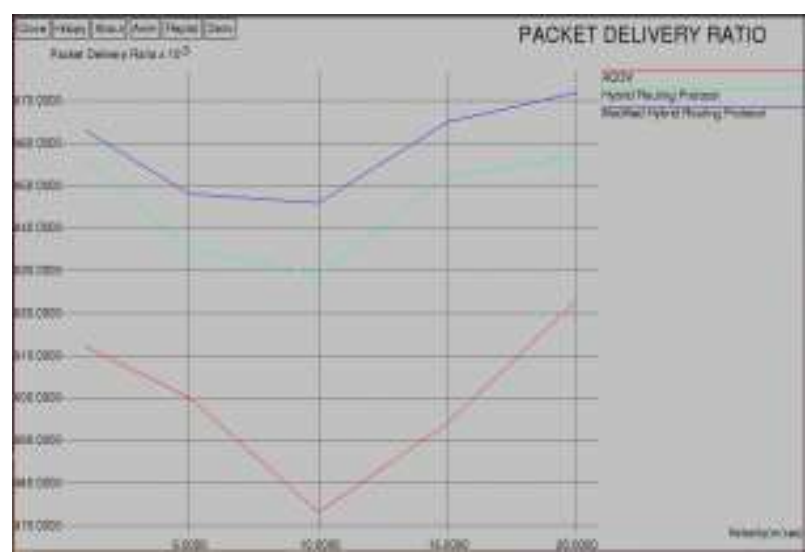

Figure.7 The packet Delivery ratio comparison among the three routing protocols for 40 nodes of the network.

For 100 Nodes

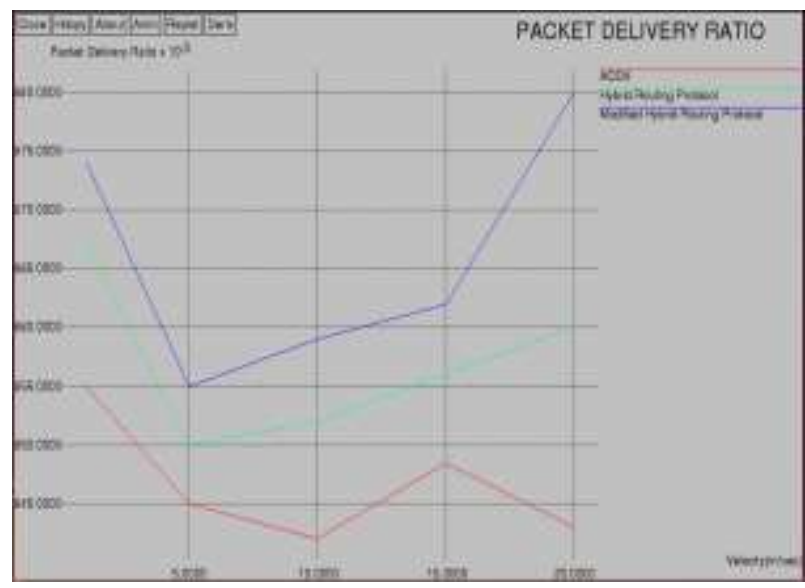

Figure8. The packet Delivery ratio comparison among the three routing protocols for 100 nodes of the network

\subsubsection{END-TO-END Delay}

End to End delay is one of the important metrics because VANET needs a small latency to transmit data packets quickly. It gives the average overall delay time taken by a packet to travel from source to destination. Delay depends on number of hops and congestion on the network. 
Only the data packets that successfully delivered to destination that counted.

$\sum$ (arrive time - send time) / $\sum$ Number of connections

\section{For 40 Nodes}

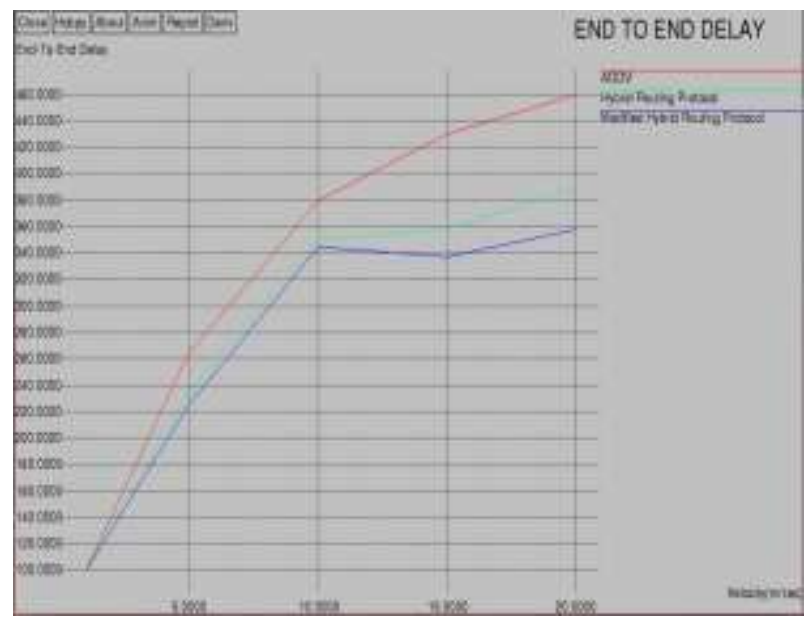

Figure.9 The end to end delay for the proposed algorithm, AODV Hybrid routing protocol for 40 nodes of the network.

\section{For 100 Nodes}

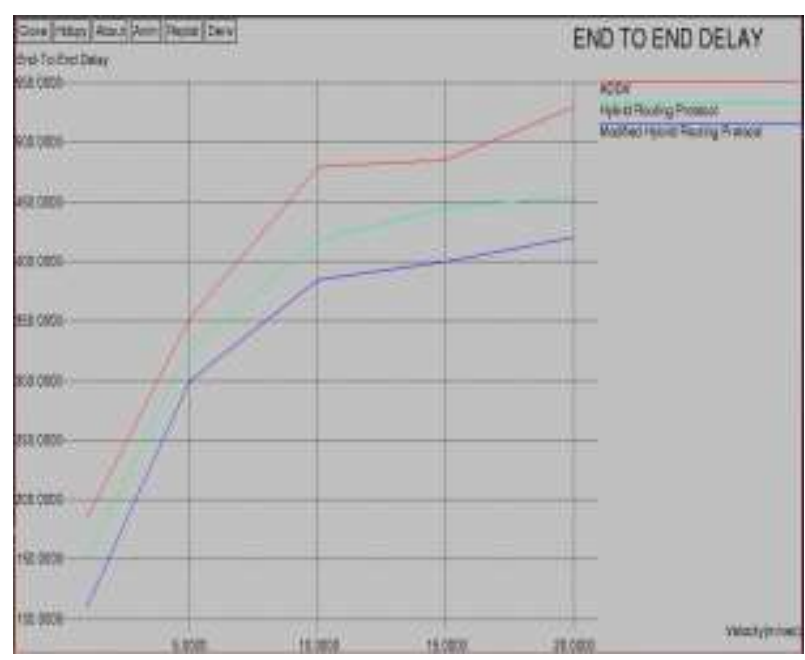

Figure.10 The end to end delay for the proposed algorithm, AODV Hybrid routing protocol for 100 nodes of the network.

\subsubsection{NORMALIZED OVERHEAD RATIO :}

The Normalized Overhead Ratio is defined as the number of routing packets required for the communication that is the total number of routing packets divided by total number of data packets delivered. This ratio is the measure of the extra packets other than the data packets generated as the percentage of the total data packets.

\section{For 40 Nodes}

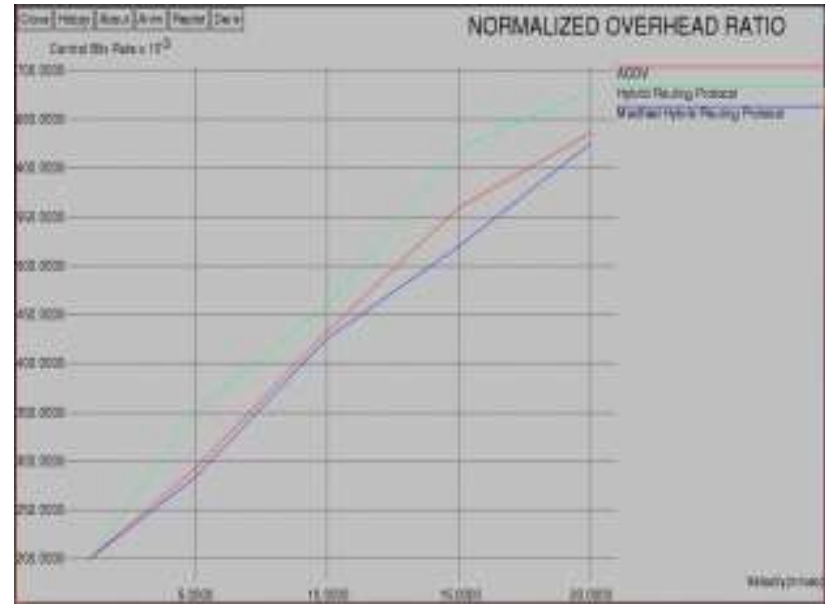

Figure.11 The Normalized overhead ratio of the three routing protocols for 40 nodes

For 100 Nodes

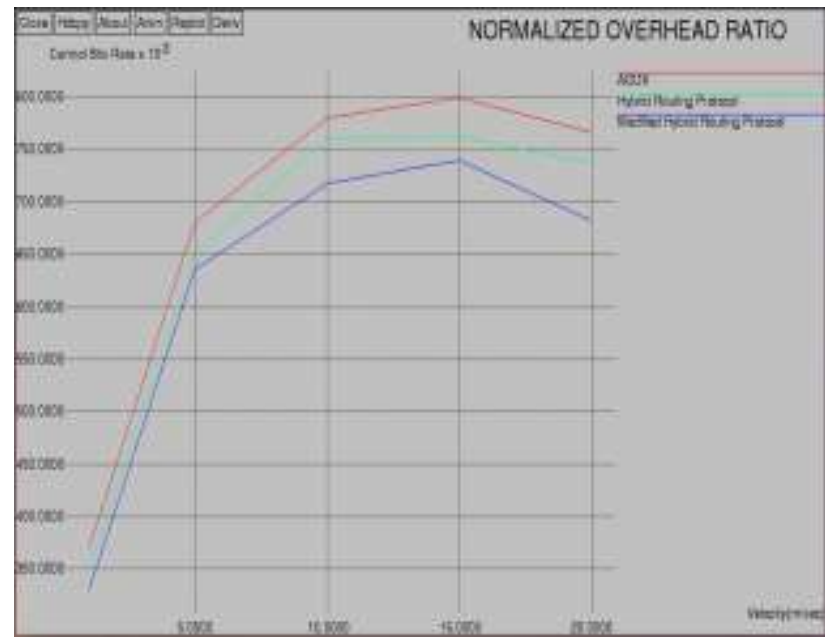

Figure.12 Normalized overhead ratio of the given routing protocols at different velocity intervals for 100 nodes of the network.

\section{CONCLUSION}

In this paper, the analysis of the proposed Hybrid algorithms is done in realistic scenario of VANET. The Realistic simulation environment is generated using the SUMO and MOVE software and the outcome of this simulation is used for network simulation using NS2. Here on comparing the performance of the introduced protocol with the Bee Swarm Routing Protocol and other static routing protocol AODV in real deployment it shows better performance and efficient results on the basis of the network parameters: end to end delay, packet delivery ratio and normalized overhead ratio. 


\section{ACKNOWLEDGMENT}

I would like to express my sincere gratitude to my advisor Er.Vinod Kumar for his valuable and constructive suggestions during the planning and development of this research work. His guidance helped me in all the time of research and writing of the thesis. Working under him was an extremely knowledgeable experience for me.

\section{REFERENCES}

[1] Siddhant Jaiswal, Dr D.S Adane, "Hybrid Approach for Routing in Vehicular Ad-hoc Network (VANET) Using Clustering Approach" International Journal of Innovative Research in Computer and Communication Engineering, Vol. 1, Issue 5, July 2013.

[2] V.S.DHAKA,RAMESH C.POONIA and LINESH RAJA, " The Realistic Mobility Evaluation of Vehicular Ad-hoc Network for Indian Automotive Networks" International Journal of Ad hoc, Sensor \& Ubiquitous Computing (IJASUC) Vol.5, No.2, April 2014.

[3] Md. Humayun Kabir, "Research Issues on Vehicular Ad hoc Network" International Journal of Engineering Trends and Technology (IJETT) - Volume 6 Number 4Dec 2013.

[4] Wiseborn Manfe Danquah,D. Turgay Altilar, "HYBRIST Mobility Model - A Novel Hybrid Mobility Model for VANET Simulations" International Journal of Computer Applications (0975 - 8887) ,Volume 86 - No 14, January 2014.

[5] Krishna Kumar Jha, Daya Sagar Baral, “ VANET Analysis for Real Time Traffic of Nepal Using SUMO and NS3 under different protocol" Department of Electronics \& Computer Engineering, Pulchowk Campus, Institute of Engineering, Tribhuvan University, Nepal.

[6] Feliz Kristianto Karnadi,Zhi Hai Mo,Kun-chan Lan, “ Rapid Generation of Realistic Mobility Models for VANET" School of Computer Science and Engineering, University of New South Wales, Sydney, NSW 2052, Australia.

[7] Nataraj B, Dr. T. Kantharaju, “ Realistic Mobility Model And Co - Operative Peer To Peer Data Transmission For VANET's Using SUMO And MOVE" IJREAT International Journal of Research in Engineering \& Advanced Technology, Volume 3, Issue 6, Dec -Jan, 2016.

[8] Michael Behrisch, Laura Bieker, Jakob Erdmann, Daniel Krajzewicz, " SUMO - Simulation of Urban MObility"Institute of Transportation Systems ,German Aerospace Center ,Rutherfordstr. 2, 12489 Berlin, Germany.

[9] http://www.isi.edu/nsnam/ns/

[10] Kun-chan Lan and Chien-Ming Chou, "Realistic Mobility Models for Vehicular Ad hoc Network (VANET) Simulations" National Cheng Kung University ,Department of Computer Science and Information Engineering ,Tainan, Taiwan.

[11] M.Sumathi, "Swarm Intelligence based Bee inspired routing protocol for multipath routing in MANET" International Journal of Advanced Research in Computer and Communication Engineering, Vol. 4, Issue 2, February 2015.
[12] K.D. Kalambe , A.R. Deshmukh, S.S Dorle, “ Particle Swarm Optimization based Routing Protocol for vehicular Ad hoc Network" International Journal of Engineering Research and General Science, ISSN 20912730, Volume 3, Issue 1, January-February, 2015.

[13] Saurabh D. Patil,D.V. Thombare,Vaishali D. Khairnar, “ DEMO: Simulation of Realistic Mobility Model and Implementation of 802.11p (DSRC) for Vehicular Networks (VANET)" International Journal of Computer Applications (0975 - 8887) Volume 43- No.21, April 2012.

[14] R.S.Raw, Vikas Toor, and N. Singh, "P ATH DURATION ANALYSIS IN VEHICULAR AD HOC NETWORK" International Journal on Ad-Hoc Networking Systems (IJANS) Vol. 2, No. 4, October 2012.

[15] Adil Baykasoùlu, Lale Özbakır and Pınar Tapkan, "Artificial Bee Colony Algorithm and Its Application to Generalized Assignment Problem" Department of Industrial Engineering, Erciyes University, Turkey.

[16] Pratik Gujar, "BIO-INSPIRED ROUTING PROTOCOL FOR VEHICULAR ADHOC NETWORKS" International Journal of Advancements in Research \& Technology, ISSN 2278-7763, Volume 3, Issue 4,April2014

[17] YUN- WEI LIN, YUH- SHYAN CHEN AND SINGLING LEE, "Routing Protocols in Vehicular Ad Hoc Networks: A Survey and Future Perspectives" Department of Computer Science and Information Engineering, National Chung Cheng University, Chia-Yi, Taiwan, R.O.C.

[18] Chitraxi Raj,Urvik Upadhayaya,Twinkle Makwana,Payal Mahida, "Simulation of VANET Using NS-3 and SUMO" International Journal of Advanced Research in Computer Science and Software Engineering, Volume 4, Issue 4, April 2014. 